\title{
APORTE DE NUTRIENTES POR FRAÇÕES DA SERAPILHEIRA EM SUCESSÃO ECOLÓGICA DE UM ECOSSISTEMA DA FLORESTA ATLÂNTICA
}

\author{
Carolina Benghi Pinto ${ }^{1}$ \\ Renato Marques ${ }^{2}$
}

\begin{abstract}
RESUMO
Com a finalidade de contribuir para o entendimento da dinâmica da ciclagem de nutrientes em ecossistema de Floresta Ombrófila Densa das Terras Baixas, na Floresta Estadual do Palmito, município de Paranaguá/PR, Brasil, foi desenvolvido este trabalho. Nele foi avaliado a deposição de fitomassa e nutrientes por frações da serapilheira em três fases de desenvolvimento da floresta: inicial (18 anos), intermediária (31 anos), e avançada (56 anos), durante o período de dois anos. Os teores de alguns nutrientes na serapilheira apresentaram variações no decorrer das estações do ano, sendo mais evidente no caso do Na e K. Observou-se um aumento nos teores de $\mathrm{N}$ e K à medida que a sucessão avança, fato associado principalmente às características morfológicas das folhas, e também ao microclima (maior umidade) e características do solo (maior espessura dos horizontes orgânicos) da fase avançada. Contrariamente, os teores de $\mathrm{Al}$ e $\mathrm{Na}$ diminuíram com o avanço da sucessão. Palavras chave: Floresta Ombrófila Densa das Terras Baixas, fases sucessionais, serapilheira
\end{abstract}

\section{NUTRIENT INPUT FROM LITTER FRACTIONS IN A ECOLOGICAL SUCESSION OF AN ATLANTIC FOREST ECOSYSTEM}

\begin{abstract}
The aim of this work was to evaluate part of the nutrient cycling dynamics in a "Floresta Ombrófila Densa das Terras Baixas" (Tropical Atlantic Forest), placed in Paranaguá, PR, Brazil. Three plots were choosed, representing the different phases of the forest regeneration: initial (18-years-old), intermediary (31-years-old), and advanced (56-years-old). The contribution of the main tree species in the releasing of nutrients to the ecosystem was evaluated from the analisys of foliar and litterfall nutrient composition. The concentration of some nutrients in litterfall showed variations during the weather seasons, being more clear for $\mathrm{Na}$ and $\mathrm{K}$. A phase effect was also observed and was represented by an increase of $\mathrm{N}$ and $\mathrm{K}$ concentrations, due specially to the morphological characteristics of leaves, and also to the local conditions - higher humidity and thicker organic soil horizon, and a decrease of $\mathrm{Al}$ and $\mathrm{Na}$ concentrations in litterfall with the developing of the forest regeneration phases.
\end{abstract}

Key-words: Floresta Ombrófila Densa das Terras Baixas, forest regeneration phases, litter

\section{INTRODUÇÃO}

A Floresta Ombrófila Densa das Terras Baixas geralmente desenvolve-se em solos arenosos, os quais apresentam baixa retenção de nutrientes devido as suas características químicas e físicas. Com isso, a vegetação tem papel fundamental na manutenção da fertilidade destes solos, através do processo denominado ciclagem de nutrientes. Nesse processo ocorre a deposição e decomposição da serapilheira, os nutrientes são liberados possibilitando o reaproveitamento pelas plantas garantindo a sustentabilidade do ecossistema.

\footnotetext{
${ }^{1}$ Mestre em Ciências Florestais - UFPR. E-mail: cabepi@ig.com.br

${ }^{2}$ Doutor em Ciências Florestais - Departamento de Solos - UFPR. E-mail: rmarques@ufpr.br
} 
Conforme Spurr \& Barnes (1982), as quantidades relativas dos diferentes nutrientes absorvidos pelas árvores, além de exercerem um papel muito importante na determinação do crescimento relativo e na capacidade competitiva das diferentes espécies, quando liberados pelo processo de decomposição, influenciam a formação das camadas superficiais do solo e, conseqüentemente, a vegetação que nele se desenvolve.

$\mathrm{Na}$ Floresta Ombrófila Densa das Terras Baixas, poucas são as informações com respeito à composição química foliar das espécies vegetais. Alguns poucos trabalhos foram desenvolvidos avaliando esse aspecto, mas em ecossistemas distintos deste (Britez, 1994). Neste ecossistema, dois trabalhos foram realizados recentemente (Boeger, 2000 e Dickow, 2000) e serviram de base para as avaliações aqui realizadas.

Neste sentido, visando complementar as informações sobre a dinâmica de ciclagem de nutrientes da Floresta Ombrófila Densa das Terras Baixas, o presente estudo teve por finalidade avaliar a contribuição de espécies arbóreas na deposição de fitomassa e liberação de nutrientes para o ecossistema em três fases sucessionais.

\section{MATERIAL E MÉTODOS}

\section{Localização e Caracterização da Área De Estudo}

A área estudada faz parte da Floresta Estadual do Palmito, localizada no município de Paranaguá - PR, entre as coordenadas $25^{\circ} 35^{\prime}$ de latitude Sul e 48 $32^{\prime}$ de longitude Oeste.

Conforme as cartas climáticas do IAPAR (1978), no município de Paranaguá, o clima predominante é o Af, segundo Köeppen, caracterizado como clima tropical super úmido, sem estação seca e isento de geadas, com temperatura média em todos os meses superiores a $18^{\circ} \mathrm{C}$, e com precipitação média do mês mais seco acima de $60 \mathrm{~mm}$.

A vegetação que recobre a área corresponde à Floresta Ombrófila Densa das Terras Baixas, e encontra-se nos mais diversos estádios sucessionais, devido a intervenções antrópicas para o cultivo, principalmente, de mandioca e abacaxi, por pelo menos 4 anos. Para este estudo foram escolhidas, três áreas, em diferentes estádios da sucessão ecológica: fase inicial, fase intermediária, fase avançada, respectivamente, com cerca de 18,31 e 56 anos, no ano 2000.

Segundo Wisniewski et al. (1997), esta área está situada na classe de solo denominada Espodossolo, com teores de areia variando entre 90 e $98 \%$, dependendo do horizonte considerado. $\mathrm{O}$ solo apresenta-se ácido em todo o perfil, com valores de $\mathrm{pH}$ da ordem de 3,2 para o horizonte A e 3,9 para o horizonte E. A saturação de bases varia entre 8 e $16 \%$ no horizonte A. Os teores de carbono são da ordem de 60g.dm-3 nas fases sucessionais inicial e avançada e da ordem de $110 \mathrm{~g}$.dm-3 na fase sucessional intermediária.

\section{Coleta e seleção de serapilheira}

Para a coleta da serapilheira foram utilizados coletores de formato quadrado, com superfície de coleta de $0,31 \mathrm{~m} 2$. Foram distribuídos aleatoriamente em número de 10 coletores/parcela de estudo (cada parcela com área de $1000 \mathrm{~m} 2$ ), e as coletas foram realizadas a cada três semanas, no período de 2 anos, a partir do outono de 1998 até o verão de 2000.

Uma vez coletada, a serapilheira foi levada ao Laboratório de Nutrição de Plantas da UFPR onde foi separada nas seguintes frações: folhas das espécies selecionadas (ES), miscelânea de folhas (espécies nãoselecionadas) (MF); ramos, flores e frutos (RFF); e outros (OT) (fração cujo estado de decomposição não permitiu a identificação do material). Nesta parte do trabalho foi avaliada somente a fração ES. As espécies que compuseram essa fração foram selecionadas com base, principalmente, na ocorrência das mesmas nos coletores e em indicadores fitossociológico (valor de importância das espécies $=$ densidade relativa + dominância relativa + freqüência relativa). Desta forma foram selecionadas as seguintes espécies: (i) Fase Inicial: Ilex theezans Mart., Ternstroemia brasiliensis Camb. e Pera glabrata (Schott) Baill; (ii) Fase Intermediária: Ilex theezans Mart., Ternstroemia brasiliensis Camb., Ocotea pulchella Mart., Clusia criuva Camb., Gomidesia schaueriana Berg e Rapanea venosa (DC.)Mez; (iii) Fase avançada: Ocotea pulchella Mart., Myrcia racemosa, Ocotea aciphylla (Nees \& Mart.) Mez e Tapirira guianensis Aubl. 
Após a separação das folhas, por espécie, estas foram colocadas para secar em estufa $\left(60^{\circ} \mathrm{C}\right)$ por um período de 72 horas, sendo a seguir determinado o seu peso de matéria seca. Para caracterização da composição química das frações, as amostras foram agrupadas por estação do ano resultando em 4 subamostras por fração. Este material foi então moído para determinação dos elementos químicos nos tecidos vegetais.

\section{Análises Químicas}

Para a determinação de $\mathrm{P}, \mathrm{K}, \mathrm{Ca}, \mathrm{Mg}$, $\mathrm{Al}, \mathrm{Na}, \mathrm{Cu}, \mathrm{Fe}, \mathrm{Mn}$ e $\mathrm{Zn}$, o material foi digerido em mufla a $500^{\circ} \mathrm{C}$ e solubilizado em $\mathrm{HCl} 10 \%$. No caso do $\mathrm{N}$, os tecidos vegetais foram digeridos em meio ácido e destilados segundo o método Kjeldahl. Os elementos $\mathrm{K}$ e $\mathrm{Na}$ foram determinados por emissão atômica; e $\mathrm{Ca}, \mathrm{Mg}$, $\mathrm{Al}, \mathrm{Cu}, \mathrm{Fe}, \mathrm{Mn}$ e $\mathrm{Zn}$ foram determinados por absorção atômica em espectrômetro 2380 Perkin-Elmer. O P foi determinado por colorimetria com vanadato-molibdato de amônio (cor amarela), sendo a leitura feita em espectrofotômetro UV/VIS 554 Perkin-Elmer.

\section{RESULTADOS E DISCUSSÃO}

$\mathrm{Na}$ figura 1 é apresentada a produção total de serapilheira, por fase sucessional e por estação climática, ao longo dos dois anos de monitoramento.

De forma geral, a fase sucessional que apresentou o maior valor médio de produção foi a fase intermediária com $7617 \mathrm{~kg} / \mathrm{ha} / \mathrm{ano}$, sendo seguida pela fase avançada com $6420 \mathrm{~kg} / \mathrm{ha} /$ ano e pela fase inicial, com $5390 \mathrm{~kg} / \mathrm{ha} /$ ano. Esses valores estão dentro da faixa dos resultados encontrados por Wisniewski (1997), na mesma área do presente estudo, e por Britez (1994), em ecossistema de restinga na Ilha do $\mathrm{Mel} / \mathrm{PR}$. As maiores taxas de queda de serapilheira ocorreram nos meses de primavera e verão, estando associadas aos períodos de maior precipitação e temperatura, e também ao efeito mecânico do vento, uma vez que quantidades significativas de folhas verdes foram encontradas nos coletores nesses períodos.

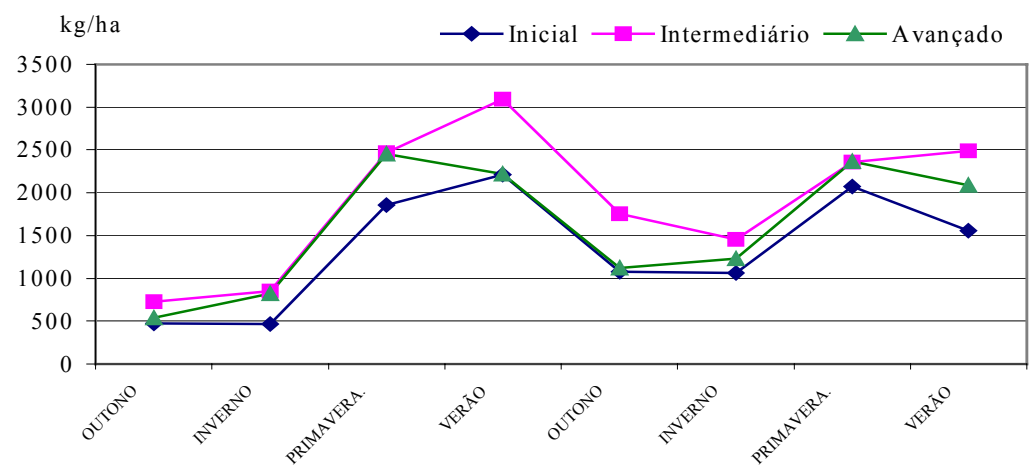

Figura 1: Dinâmica de deposição de serapilheira, por fase sucessionail, ao longo das estações durante os dois anos de estudo

Figure1: Litterfall dinamics in forest regeneration phases, through the seasons of the year

A produção de serapilheira por espécie nas diferentes fases sucessionais é apresentada na tabela 1. Observa-se que, à medida que a sucessão vegetal evolui, a participação das espécies na produção de serapilheira torna-se menos desequilibrada.

Nas fases inicial e intermediária Ilex theezans Mart. foi a espécie que apresentou a maior participação, representando, respectivamente, $67 \%$ e $30 \%$ do total de serapilheira produzida. Isso está certamente ligado ao valor de importância (VI) desta espécie, nestas duas fases, o qual é destacadamente superior aos das demais espécies, por ser espécie tipicamente pioneira (Wisniewski, 1997). Conforme Vitousek (1984), em geral, espécies pioneiras, crescem bem sobre solos de baixa fertilidade, pois apresentam uma elevada eficiência no uso de nutrientes escassos no ecossistema. 
Na faz' avançada também observou-se que a maior participação na deposição de serapilheira foi das espécies com os maiores valores de importância. Mas, nesse caso, não existe uma espécie que se destaca, pois a composição florística é representada por um maior número de espécies, com VIs mais equilibrados.

Tabela 1: Produção média anual de serapilheira e valor de importância (vi) das espécies, nas diferentes fases sucessionais

Table 1: Anual average of litter production and importance value of the species, in the diferent forest regeneration phases.

\begin{tabular}{|c|c|c|c|c|c|c|c|c|}
\hline INICIAL & MÉDIA (kg/ha) & VI $(\%)$ & INTERMEDIÁRIA & $\begin{array}{l}\text { MÉDIA } \\
(\mathrm{kg} / \mathrm{ha})\end{array}$ & VI $(\%)$ & AVANÇADA & $\begin{array}{l}\text { MÉDIA } \\
(\mathrm{kg} / \mathrm{ha})\end{array}$ & $\begin{array}{l}\text { VI } \\
(\%)\end{array}$ \\
\hline Ilex theezans & $\begin{array}{l}3620,5 b^{*} \\
(297,3)^{* *}\end{array}$ & 159,56 & Ilex theezans & $\begin{array}{c}2361,5 \mathrm{~b} \\
(242,3)\end{array}$ & 72,88 & Ocotea pulchella & $\begin{array}{c}362,7 \mathrm{~b} \\
(10,7)\end{array}$ & 29,08 \\
\hline $\begin{array}{c}\text { Ternstroemia } \\
\text { brasiliensis }\end{array}$ & $\begin{array}{c}361,0 \mathrm{a} \\
(36,6)\end{array}$ & 29,24 & Ternstroemia brasiliensis & $\begin{array}{c}406,5 \mathrm{a} \\
(74,8)\end{array}$ & 31,65 & Ocotea aciphylla & $\begin{array}{c}711,9 \mathrm{c} \\
(63,4)\end{array}$ & 16,56 \\
\hline \multirow[t]{4}{*}{ Pera glabrata } & $\begin{array}{c}146,7 \mathrm{a} \\
(22,5)\end{array}$ & 15,52 & Ocotea pulchella & $\begin{array}{c}352,8 \mathrm{a} \\
(35,3)\end{array}$ & 13,25 & Tapirira guianensis & $\begin{array}{l}986,2 \mathrm{~d} \\
(114,7)\end{array}$ & 29,08 \\
\hline & & & Clusia criuva & $\begin{array}{c}337,9 \mathrm{a} \\
(15,6)\end{array}$ & 14,41 & Myrcia racemosa & $\begin{array}{c}151,8 \mathrm{a} \\
(5,3)\end{array}$ & 12,46 \\
\hline & & & Rapanea venosa & $\begin{array}{c}476,2 \mathrm{a} \\
(42,2)\end{array}$ & 29,99 & & & \\
\hline & & & Gomidesia schaueriana & $\begin{array}{c}332,2 \mathrm{a} \\
(44,8)\end{array}$ & 29,65 & & & \\
\hline
\end{tabular}

* Valores em colunas seguidos de mesma letra não diferem entre si pelo teste de Fischer's protected LSD ao nível de 5\% .

**(Desvio padrão)

Na tabela 2 podem ser observados os teores médios de $\mathrm{N}, \mathrm{P}, \mathrm{K}, \mathrm{Ca}, \mathrm{Mg}$, $\mathrm{Na}$ e $\mathrm{Al}$, nos tecidos foliares para as espécies das três fases sucessionais. De maneira geral, a ordem decrescente de concentração dos elementos observada nos tecidos foliares foi a seguinte: $\mathrm{N}>\mathrm{Ca}>\mathrm{Mg}>\mathrm{K}>\mathrm{Al}$ e $\mathrm{P}>\mathrm{Na}$, a qual assemelha-se às seqüências constatadas em outros estudos realizados em restingas (Britez, 1994; Moraes, 1993).

Tabela 2: Teores médios de macronutrientes, sódio e alumínio na serapilheira para as espécies selecionadas nas três fases sucessionais

Table 2: $\quad$ Average concentrations of macronutrients, sodium and aluminium in the litter for the selected species in the three forest regeneration phases.

\begin{tabular}{|c|c|c|c|c|c|c|c|}
\hline FASES & $\mathrm{N}$ & $\mathrm{P}$ & $\mathrm{K}$ & $\mathrm{Ca}$ & $\mathrm{Mg}$ & $\mathrm{Na}$ & $\mathrm{Al}$ \\
\hline & \multicolumn{7}{|c|}{$\mathrm{g} / \mathrm{kg}$} \\
\hline \multicolumn{8}{|l|}{ INICIAL } \\
\hline Ilex theezans & $\begin{array}{c}5,56 \mathrm{a}^{*} \\
(1,84)^{* *}\end{array}$ & $\begin{array}{l}0,32 \mathrm{a} \\
(0,14)\end{array}$ & $\begin{array}{l}0,99 \mathrm{a} \\
(0,12)\end{array}$ & $\begin{array}{c}11,78 \mathrm{a} \\
(1,77)\end{array}$ & $\begin{array}{l}5,38 \mathrm{~b} \\
(0,28)\end{array}$ & $\begin{array}{l}0,16 \mathrm{a} \\
(0,03)\end{array}$ & $\begin{array}{l}0,46 \mathrm{a} \\
(0,08)\end{array}$ \\
\hline Ternstroemia brasiliensis & $\begin{array}{l}5,58 \mathrm{a} \\
(1,67)\end{array}$ & $\begin{array}{l}0,34 \mathrm{a} \\
(0,10)\end{array}$ & $\begin{array}{l}1,21 \mathrm{a} \\
(0,44)\end{array}$ & $\begin{array}{c}10,41 \mathrm{a} \\
(0,89)\end{array}$ & $\begin{array}{l}3,51 \mathrm{a} \\
(0,11)\end{array}$ & $\begin{array}{l}0,12 \mathrm{~b} \\
(0,02)\end{array}$ & $\begin{array}{l}1,57 \mathrm{~b} \\
(0,43)\end{array}$ \\
\hline Pera glabrata & $\begin{array}{c}20,38 \mathrm{~b} \\
(1,39)\end{array}$ & $\begin{array}{l}1,44 \mathrm{~b} \\
(0,26)\end{array}$ & $\begin{array}{l}2,24 \mathrm{~b} \\
(0,48)\end{array}$ & $\begin{array}{c}19,80 \mathrm{~b} \\
(2,95)\end{array}$ & $\begin{array}{l}7,20 \mathrm{c} \\
(1,46)\end{array}$ & $\begin{array}{l}0,21 \mathrm{c} \\
(0,04)\end{array}$ & $\begin{array}{l}0,23 \mathrm{a} \\
(0,08)\end{array}$ \\
\hline \multicolumn{8}{|l|}{ INTERMEDIÁRIA } \\
\hline Ilex theezans & $\begin{array}{c}10,15 \mathrm{c} \\
(2,70)\end{array}$ & $\begin{array}{c}0,35 \mathrm{~b} \mathrm{c} \\
(0,11)\end{array}$ & $\begin{array}{l}1,95 \mathrm{c} \\
(0,52)\end{array}$ & $\begin{array}{l}7,62 \mathrm{~b} \\
(0,88)\end{array}$ & $\begin{array}{l}3,74 \mathrm{~d} \\
(0,40)\end{array}$ & $\begin{array}{l}0,18 \mathrm{e} \\
(0,04)\end{array}$ & $\begin{array}{l}0,35 \mathrm{~b} \\
(0,08)\end{array}$ \\
\hline Ternstroemia brasiliensis & $\begin{array}{l}7,40 \mathrm{a} \\
(0,74)\end{array}$ & $\begin{array}{c}0,24 \mathrm{a} \mathrm{b} \\
(0,10)\end{array}$ & $\begin{array}{c}1,78 \mathrm{~b} \mathrm{c} \\
(0,32)\end{array}$ & $\begin{array}{l}7,97 \mathrm{~b} \\
(0,39)\end{array}$ & $\begin{array}{l}4,12 \mathrm{e} \\
(0,37)\end{array}$ & $\begin{array}{l}0,15 \mathrm{~d} \\
(0,03)\end{array}$ & $\begin{array}{l}1,31 \mathrm{c} \\
(0,20)\end{array}$ \\
\hline Ocotea pulchella & $\begin{array}{c}9,76 \mathrm{~b} c \\
(1,69)\end{array}$ & $\begin{array}{l}0,40 \mathrm{c} \\
(0,11)\end{array}$ & $\begin{array}{l}1,19 \mathrm{a} \\
(0,22)\end{array}$ & $\begin{array}{l}5,73 \mathrm{a} \\
(0,70)\end{array}$ & $\begin{array}{l}1,78 \mathrm{a} \\
(0,07)\end{array}$ & $\begin{array}{c}0,08 \mathrm{a} \mathrm{b} \\
(0,02)\end{array}$ & $\begin{array}{l}0,45 \mathrm{~b} \\
(0,08)\end{array}$ \\
\hline Clusia criuva & $\begin{array}{l}6,84 \mathrm{a} \\
(1,98)\end{array}$ & $\begin{array}{c}0,29 \mathrm{a} \mathrm{b} \mathrm{c} \\
(0,14)\end{array}$ & $\begin{array}{c}1,81 \mathrm{~b} \mathrm{c} \\
(0,21)\end{array}$ & $\begin{array}{c}13,44 \mathrm{~d} \\
(0,52)\end{array}$ & $\begin{array}{l}3,29 \mathrm{c} \\
(0,14)\end{array}$ & $\begin{array}{l}0,11 \mathrm{c} \\
(0,01)\end{array}$ & $\begin{array}{l}0,08 \mathrm{a} \\
(0,03)\end{array}$ \\
\hline Rapanea venosa & $\begin{array}{c}8,13 \mathrm{a} b \\
(1,31)\end{array}$ & $\begin{array}{l}0,22 \mathrm{a} \\
(0,09)\end{array}$ & $\begin{array}{l}1,35 \mathrm{a} \\
(0,16)\end{array}$ & $\begin{array}{l}9,72 \mathrm{c} \\
(1,15)\end{array}$ & $\begin{array}{l}2,47 b \\
(0,17)\end{array}$ & $\begin{array}{c}0,13 \mathrm{c} \mathrm{d} \\
(0,02)\end{array}$ & $\begin{array}{l}0,11 \mathrm{a} \\
(0,03)\end{array}$ \\
\hline Gomidesia schaueriana & $\begin{array}{l}7,30 \mathrm{a} \\
(1,54) \\
\end{array}$ & $\begin{array}{c}0,28 \mathrm{a} \mathrm{b} \mathrm{c} \\
(0,12)\end{array}$ & $\begin{array}{c}1,48 \mathrm{a} b \\
(0,33)\end{array}$ & $\begin{array}{l}6,52 \mathrm{a} \\
(0,76) \\
\end{array}$ & $\begin{array}{l}2,48 \mathrm{~b} \\
(0,37) \\
\end{array}$ & $\begin{array}{c}0,11 \mathrm{~b} \mathrm{c} \\
(0,03)\end{array}$ & $\begin{array}{l}0,13 \mathrm{a} \\
(0,04) \\
\end{array}$ \\
\hline \multicolumn{8}{|l|}{ AVANÇADA } \\
\hline Ocotea pulchella & $\begin{array}{c}10,19 \mathrm{a} \\
(2,56)\end{array}$ & $\begin{array}{c}0,43 \mathrm{a} \mathrm{b} \\
(0,16)\end{array}$ & $\begin{array}{l}1,28 \mathrm{a} \\
(0,31)\end{array}$ & $\begin{array}{l}6,73 \mathrm{a} \\
(0,48)\end{array}$ & $\begin{array}{l}1,81 \mathrm{a} \\
(0,11)\end{array}$ & $\begin{array}{l}0,06 \mathrm{~b} \\
(0,02)\end{array}$ & $\begin{array}{l}0,34 \mathrm{c} \\
(0,05)\end{array}$ \\
\hline Ocotea aciphylla & $\begin{array}{c}11,59 \mathrm{a} b \\
(1,39)\end{array}$ & $\begin{array}{l}0,32 \mathrm{a} \\
(0,10)\end{array}$ & $\begin{array}{l}1,77 \mathrm{~b} \\
(0,35)\end{array}$ & $\begin{array}{l}7,50 \mathrm{a} \\
(0,68)\end{array}$ & $\begin{array}{l}2,05 \mathrm{~b} \\
(0,12)\end{array}$ & $\begin{array}{c}0,05 \mathrm{a} \mathrm{b} \\
(0,01)\end{array}$ & $\begin{array}{l}0,09 \text { a } \\
(0,03)\end{array}$ \\
\hline Tapirira guianensis & $\begin{array}{c}10,86 \mathrm{a} \mathrm{b} \\
(2,16)\end{array}$ & $\begin{array}{l}0,36 \mathrm{a} \\
(0,14)\end{array}$ & $\begin{array}{l}1,96 \mathrm{bc} \\
(0,28))\end{array}$ & $\begin{array}{c}12,53 \mathrm{~b} \\
(1,22)\end{array}$ & $\begin{array}{l}2,32 \mathrm{c} \\
(0,18)\end{array}$ & $\begin{array}{l}0,04 \mathrm{a} \\
(0,01)\end{array}$ & $\begin{array}{l}0,09 \mathrm{a} \\
(0,04)\end{array}$ \\
\hline Myrcia racemosa & $\begin{array}{c}12,51 \mathrm{~b} \\
(1,47)\end{array}$ & $\begin{array}{l}0,49 \mathrm{~b} \\
(0,07)\end{array}$ & $\begin{array}{l}2,15 \mathrm{c} \\
(0,35)\end{array}$ & $\begin{array}{c}16,89 \mathrm{c} \\
(0,99)\end{array}$ & $\begin{array}{l}2,97 \mathrm{~d} \\
(0,39)\end{array}$ & $\begin{array}{c}0,05 \mathrm{a} \mathrm{b} \\
(0,01)\end{array}$ & $\begin{array}{l}0,15 \mathrm{~b} \\
(0,05)\end{array}$ \\
\hline
\end{tabular}

* Valores em colunas seguidos de mesma letra não diferem entre si pelo teste de Fischer's protected LSD ao nível de 5\% .**(Desvio padrão) 
No que diz respeito à evolução da sucessão observa-se que para a maioria das espécies ocorreu uma tendência de aumento nos teores de $\mathrm{N}$ e $\mathrm{K}$, fato associado às características morfológicas das folhas, condições do solo e microclima. À medida que a sucessão evolui, as folhas apresentam menor esclerofilia, e os horizontes orgânicos tornamse mais espessos, favorecendo o estoque de água e nutrientes. O microclima torna-se mais úmido, o que favorece a decomposição do material orgânico e reaproveitamento dos nutrientes pelas plantas. Já os teores de $\mathrm{Na}$ e $\mathrm{Al}$ diminuem consideravelmente à medida que a sucessão evolui, o mesmo, mas não tão evidente, ocorrendo para o $\mathrm{Mg}$. Os demais nutrientes não apresentaram uma tendência clara ao longo da sucessão.

As espécies que mais destacaram-se com relação aos teores de macronutrientes foram Pera glabrata (Schott) Baill da fase inicial e Myrcia racemosa da fase avançada, as quais apresentaram as maiores concentrações, devido, possivelmente, às características das folhas destas espécies, que apresentam baixo escleromorfismo.

$\mathrm{Na}$ tabela 3 são apresentados os teores de micronutrientes nos tecidos foliares das espécies por fase sucessional. Na fase inicial, Ilex theezans Mart. apresenta o maior teor de Mn, e Pera glabrata (Schott) Baill os maiores teores dos demais nutrientes. $\mathrm{Na}$ fase intermediária, nota-se que para $\mathrm{Fe}, \mathrm{Mn}$ e $\mathrm{Zn}$ os maiores valores ocorreram para Clusia criuva Camb., e que para $\mathrm{Cu}$ os maiores valores apareceram para Gomidesia schaueriana Berg. $\mathrm{Na}$ fase avançada observou-se que, para $\mathrm{Cu}$ e $\mathrm{Mn}$, a espécie que apresentou o maior valor foi Myrcia racemosa, e para o $\mathrm{Zn}$ o maior valor foi para Ocotea aciphylla (Nees) Mez. De maneira geral, para a maioria das espécies, a ordem decrescente de concentração dos micronutrientes, observada nos tecidos foliares, foi a seguinte: $\mathrm{Mn}>\mathrm{Fe}>\mathrm{Zn}>\mathrm{Cu}$, a qual segue a tendência verificada por Moraes (1993). Não foi observado um comportamento claro da concentração dos micronutrientes ao longo da sucessão.

Tabela 3: Teores médios de micronutrientes na serapilheira para as espécies selecionadas nas três fases sucessionais

Table 3: Average micronutrient concentrations of the litter for the selected species in the three forest regeneration phases

\begin{tabular}{|c|c|c|c|c|}
\hline FASES & $\mathrm{Mn}$ & $\mathrm{Fe}$ & $\mathrm{Zn}$ & $\mathrm{Cu}$ \\
\hline & \multicolumn{4}{|c|}{$\mathrm{mg} / \mathrm{kg}$} \\
\hline \multicolumn{5}{|l|}{ INICIAL } \\
\hline Ilex theezans & $\begin{array}{l}2742,03 b^{*} \\
(839,62)^{* *}\end{array}$ & $\begin{array}{l}77,31 \mathrm{a} \\
(21,77)\end{array}$ & $\begin{array}{c}29,11 \mathrm{~b} \\
(5,27)\end{array}$ & $\begin{array}{l}5,56 \mathrm{a} \\
(0,78)\end{array}$ \\
\hline Ternstroemia brasiliensis & $\begin{array}{l}38,92 \mathrm{a} \\
(15,76)\end{array}$ & $\begin{array}{l}81,12 \mathrm{a} \\
(14,01)\end{array}$ & $\begin{array}{l}8,84 \mathrm{a} \\
(1,57)\end{array}$ & $\begin{array}{l}4,85 \mathrm{a} \\
(1,34)\end{array}$ \\
\hline Pera glabrata & $\begin{array}{c}438,42 \mathrm{a} \\
(48,99) \\
\end{array}$ & $\begin{array}{c}142,33 \mathrm{~b} \\
(35,49) \\
\end{array}$ & $\begin{array}{c}39,22 \mathrm{c} \\
(5,67)\end{array}$ & $\begin{array}{c}22,39 \mathrm{~b} \\
(3,45)\end{array}$ \\
\hline \multicolumn{5}{|l|}{ INTERMEDIÁRIA } \\
\hline Ilex theezans & $\begin{array}{c}407,4 \mathrm{~b} \\
(643,94)\end{array}$ & $\begin{array}{l}81,01 \text { a } \\
(17,45)\end{array}$ & $\begin{array}{c}18,97 \mathrm{c} \\
(2,58)\end{array}$ & $\begin{array}{l}8,13 \mathrm{a} \\
(0,90)\end{array}$ \\
\hline Ternstroemia brasiliensis & $\begin{array}{l}35,64 \mathrm{a} \\
(12,39)\end{array}$ & $\begin{array}{l}71,59 \mathrm{a} \\
(17,23)\end{array}$ & $\begin{array}{l}9,98 \mathrm{a} \\
(1,53)\end{array}$ & $\begin{array}{l}6,56 \mathrm{a} \\
(1,51)\end{array}$ \\
\hline Ocotea pulchella & $\begin{array}{c}153,65 \mathrm{a} \\
(30,21)\end{array}$ & $\begin{array}{l}90,30 \mathrm{a} \\
(21,38)\end{array}$ & $\begin{array}{c}14,41 \mathrm{~b} \\
(2,99)\end{array}$ & $\begin{array}{l}6,13 \mathrm{a} \\
(1,67)\end{array}$ \\
\hline Clusia criuva & $\begin{array}{c}2069,86 \mathrm{c} \\
(33,17)\end{array}$ & $\begin{array}{c}75,70 \mathrm{a} \\
(113,19)\end{array}$ & $\begin{array}{c}28,08 \mathrm{~d} \\
(2,79)\end{array}$ & $\begin{array}{l}6,84 \mathrm{a} \\
(2,26)\end{array}$ \\
\hline Rapanea venosa & $\begin{array}{c}135,14 \mathrm{a} \\
(20,15)\end{array}$ & $\begin{array}{l}87,25 \mathrm{a} \\
(21,28)\end{array}$ & $\begin{array}{c}11,98 \mathrm{ab} \\
(1,73)\end{array}$ & $\begin{array}{l}7,27 \mathrm{a} \\
(2,21)\end{array}$ \\
\hline Gomidesia schaueriana. & $\begin{array}{c}157,29 \mathrm{a} \\
(25,79)\end{array}$ & $\begin{array}{l}96,90 \mathrm{a} \\
(18,38) \\
\end{array}$ & $\begin{array}{c}11,42 \mathrm{a} \\
(1,81)\end{array}$ & $\begin{array}{c}13,13 \mathrm{~b} \\
(2,54)\end{array}$ \\
\hline \multicolumn{5}{|l|}{ AVANÇADA } \\
\hline Ocotea pulchella & $\begin{array}{c}177,94 \mathrm{~b} \\
(37,47)\end{array}$ & $\begin{array}{c}82,85 \mathrm{ab} \\
(10,26)\end{array}$ & $\begin{array}{c}13.97 \\
(3,41)\end{array}$ & $\begin{array}{l}5,84 \mathrm{a} \\
(2,19)\end{array}$ \\
\hline Ocotea aciphylla & $\begin{array}{c}232,01 \mathrm{~b} \\
(43,29)\end{array}$ & $\begin{array}{c}80,03 \mathrm{ab} \\
(13,98)\end{array}$ & $\begin{array}{c}19,51 \mathrm{~b} \\
(1,90)\end{array}$ & $\begin{array}{l}5,98 \mathrm{a} \\
(2,00)\end{array}$ \\
\hline Tapirira guianensis & $\begin{array}{c}102,29 \mathrm{a} \\
(50,07)\end{array}$ & $\begin{array}{l}84,39 \mathrm{~b} \\
(20,14)\end{array}$ & $\begin{array}{c}15,68 \mathrm{a} \\
(1,49)\end{array}$ & $\begin{array}{l}5,70 \mathrm{a} \\
(1,97)\end{array}$ \\
\hline Myrcia racemosa & $\begin{array}{l}378,57 \mathrm{c} \\
(104,86)\end{array}$ & $\begin{array}{c}111,53 \mathrm{c} \\
(27,23)\end{array}$ & $\begin{array}{c}15,59 \mathrm{a} \\
(2,49)\end{array}$ & $\begin{array}{l}5,99 \mathrm{a} \\
(1,63)\end{array}$ \\
\hline
\end{tabular}

* Valores em colunas seguidos de mesma letra não diferem entre si pelo teste de Fischer's protected LSD ao nível de 5\% **(Desvio padrão) 

sucessioal.

A tabela 4 demonstra as quantidades de nutrientes em $\mathrm{kg} / \mathrm{ha}$ por espécie em cada fase

Tabela 4: Quantidade média de macronutrientes, sódio e alumínio na serapilheira para as espécies selecionadas nas três fases sucessionais

Table 4: Average quantity of macronutrients, sodium and aluminium in the litter for the selected species in the

\begin{tabular}{|c|c|c|c|c|c|c|c|}
\hline FASES & $\mathrm{N}$ & $\mathrm{P}$ & $\mathrm{K}$ & $\mathrm{Ca}$ & $\mathrm{Mg}$ & $\mathrm{Na}$ & $\mathrm{Al}$ \\
\hline & \multicolumn{7}{|c|}{$\mathrm{kg} / \mathrm{há}$} \\
\hline \multicolumn{8}{|l|}{ INICIAL } \\
\hline Ilex theezans & 20,129 & 1,159 & 3,584 & 42,649 & 19,478 & 0,579 & 1,665 \\
\hline Ternstroemia brasiliensis & 2,014 & 0,123 & 0,437 & 3,758 & 1,267 & 0,043 & 0,567 \\
\hline Pera glabrata & 2,990 & 0,210 & 0,329 & 2,904 & 1,056 & 0,031 & 0,034 \\
\hline \multicolumn{8}{|l|}{ INTERMEDIÁRIA } \\
\hline Ilex theezans & 23,965 & 0,827 & 4,604 & 17,994 & 8,832 & 0,425 & 0,826 \\
\hline Ternstroemia brasiliensis & 3,008 & 0,097 & 0,724 & 3,240 & 1,674 & 0,060 & 0,533 \\
\hline Ocotea pulchella & 3,443 & 0,141 & 0,420 & 2,022 & 0,627 & 0,028 & 0,353 \\
\hline Clusia criuva & 2,308 & 0,097 & 0,611 & 4,541 & 1,112 & 0,037 & 0,338 \\
\hline Rapanea venosa & 3,871 & 0,105 & 0,643 & 4,629 & 1,176 & 0,062 & 0,514 \\
\hline Gomidesia schaueriana & 2,425 & 0,093 & 0,492 & 2,166 & 0,823 & 0,036 & 0,043 \\
\hline \multicolumn{8}{|l|}{ AVANÇADA } \\
\hline Ocotea pulchella & 3,695 & 0,155 & 0,464 & 2,440 & 0,362 & 0,022 & 0,123 \\
\hline Ocotea aciphylla & 11,148 & 0,228 & 1,260 & 5,339 & 1,459 & 0,036 & 0,064 \\
\hline Tapirira guianensis & 16,094 & 0,355 & 1,932 & 12,357 & 2,288 & 0,039 & 0,089 \\
\hline Myrcia racemosa & 2,095 & 0,074 & 0,326 & 2,564 & 0,450 & 0,008 & 0,023 \\
\hline
\end{tabular}

Na tabela 5 é apresentada a contribuição percentual de cada espécie estudada para produção de serapilheira e para a deposição de nutrientes na forma de matéria seca.

Tabela 5: Contribuição das espécies para a produção de serapilheira e deposição de macronutrientes, sódio e alumínio nas fases sucessionais

Table 5: Species contribution for litter production and deposition of macronutrient, sodium and aluminium in

\begin{tabular}{|c|c|c|c|c|c|c|c|c|}
\hline FASES & PESO & $\mathrm{N}$ & $\mathrm{P}$ & $\mathrm{K}$ & $\mathrm{Ca}$ & $\mathrm{Mg}$ & $\mathrm{Na}$ & $\mathrm{Al}$ \\
\hline \multicolumn{9}{|c|}{$\%$} \\
\hline \multicolumn{9}{|l|}{ INICIAL } \\
\hline Ilex theezans & 87,7 & 80 & 78 & 83 & 86 & 89 & 7 & 73 \\
\hline Ternstroemia brasiliensis & 8,7 & 8 & 8 & 10 & 8 & 6 & 7 & 25 \\
\hline Pera glabrata & 3,5 & 12 & 14 & 7 & 6 & 5 & 5 & 2 \\
\hline
\end{tabular}

\begin{tabular}{|c|c|c|c|c|c|c|c|c|}
\hline Ilex theezans & 55,3 & 61 & 61 & 60 & 52 & 62 & 66 & 32 \\
\hline $\begin{array}{l}\text { Ternstroemia brasiliensis } \\
\text { Ocotea pulchella }\end{array}$ & $\begin{array}{l}9,5 \\
8,3\end{array}$ & $\begin{array}{l}8 \\
9\end{array}$ & $\begin{array}{c}7 \\
10\end{array}$ & $\begin{array}{c}10 \\
6\end{array}$ & $\begin{array}{l}9 \\
6\end{array}$ & $\begin{array}{c}12 \\
4\end{array}$ & $\begin{array}{l}9 \\
4\end{array}$ & $\begin{array}{l}20 \\
13\end{array}$ \\
\hline $\begin{array}{c}\text { Clusia criuva } \\
\text { Rapanea venosa } \\
\text { Gomidesia schaueriana }\end{array}$ & $\begin{array}{c}7,9 \\
11,2 \\
7,8 \\
\end{array}$ & $\begin{array}{c}6 \\
10 \\
6 \\
\end{array}$ & $\begin{array}{l}7 \\
7 \\
7 \\
\end{array}$ & $\begin{array}{l}8 \\
9 \\
7\end{array}$ & $\begin{array}{c}13 \\
13 \\
6 \\
\end{array}$ & $\begin{array}{l}8 \\
8 \\
6 \\
\end{array}$ & $\begin{array}{l}6 \\
9 \\
6 \\
\end{array}$ & $\begin{array}{c}13 \\
20 \\
2\end{array}$ \\
\hline AVANÇADA & & & & & & & & \\
\hline $\begin{array}{l}\text { Ocotea pulchella } \\
\text { Ocotea aciphylla } \\
\text { Tapirira guianensis } \\
\text { Myrcia racemosa }\end{array}$ & $\begin{array}{c}16,4 \\
32,2 \\
44,6 \\
6,9 \\
\end{array}$ & $\begin{array}{c}11 \\
34 \\
49 \\
6 \\
\end{array}$ & $\begin{array}{c}19 \\
28 \\
44 \\
9 \\
\end{array}$ & $\begin{array}{c}12 \\
32 \\
48 \\
8 \\
\end{array}$ & $\begin{array}{l}11 \\
24 \\
54 \\
11\end{array}$ & $\begin{array}{c}8 \\
32 \\
50 \\
10\end{array}$ & $\begin{array}{c}21 \\
34 \\
38 \\
7 \\
\end{array}$ & $\begin{array}{c}41 \\
22 \\
30 \\
7\end{array}$ \\
\hline
\end{tabular}

Pode ser observado que, de uma forma geral, as espécies que contribuíram com maior percentual de produção de matéria seca foram também as que aportaram maior percentual de nutrientes ao ecossistema. Esse é o caso da Ilex theezans Mart., das fases inicial e intermediária que apesar, dos baixos teores de nutrientes em seus tecidos, apresentou maior deposição de nutrientes por hectare.

Algumas espécies apresentaram pouca participação em termos de aporte de matéria seca, mas em relação ao aporte de nutrientes essas espécies apresentam uma contribuição relativamente alta. Esse é o caso da Pera glabrata (Schott) Baill, na fase inicial, no que concerne $\mathrm{N}$ e $\mathrm{P}$. Esta espécie produziu menos 
da metade do percentual de matéria seca produzido pela Terstroemia brasiliensis Camb., mas depositou cerca de duas vezes mais $\mathrm{N}$ e $\mathrm{P}$ sobre o solo.

$\mathrm{Na}$ fase intermediária, com exceção da Ilex theezans Mart., tanto a produção de serapilheira, como a deposição de nutrientes por hectare ocorreu de forma mais equilibrada entre as espécies.

$\mathrm{Na}$ fase avançada, Myrcia racemosa e Ocotea pulchella Mart. foram espécies que Destacaram-se por apresentar elevados teores nos tecidos foliares, mas que contribuíram pouco na deposição de serapilheira. Já Tapirira guianensis Aubl. e Ocotea aciphylla (Nees) Mez desta fase apresentaram comportamento semelhante ao de Ilex theezans Mart., apresentando uma grande participação na produção de serapilheira. Esse fato sugere uma estratégia do ecossistema no sentido de compensar a baixa concentração de nutrientes nos tecidos foliares pela elevada produção de fitomassa.

\section{CONCLUSÕES}

- As espécies que apresentaram os maiores valores de importância foram as espécies que mais contribuíram na produção de serapilheira, sendo que Ilex theezans Mart., nas fases inicial e intermediária, e Tapirira guianensis Aubl. na fase avançada foram as que mais participaram na produção.

- As espécies que menos participaram na produção de serapilheira foram as que apresentaram as maiores concentrações nos tecidos foliares para a maioria dos nutrientes, já as espécies com maior contribuição na produção de serapilheira foram as que apresentaram as menores concentrações. Esse aspecto esteve relacionado as características morfológicas das folhas, sendo que as espécies que apresentaram os maiores teores de nutrientes são as que possuem menor escleromorfismo.

- O aumento dos teores de alguns nutrientes, como $\mathrm{N}$ e $\mathrm{K}$ da serapilheira, à medida que aumenta o gradiente sucessional, além de estar relacionado às características morfológicas das folhas, está associado ao acúmulo de serapilheira sobre o solo. Já a causa da diminuição de nutrientes, como o $\mathrm{Na}$ ao longo do gradiente sucessional, requer maiores investigações.

- A variação nos teores de nutrientes na serapilheira, ao longo das estações, indica a influência de aspectos climáticos como a precipitação, principalmente no caso do $\mathrm{Na}$ e $\mathrm{K}$, e da translocação dos nutrientes nos tecidos foliares, conforme às necessidades fisiológicas das plantas durante determinada estação climática.

\section{REFERÊNCIAS}

BRITEZ, R. M. Ciclagem de nutrientes minerais em duas florestas da planície litorânea da Ilha do Mel, Paranaguá, PR. Curitiba, 1994. 240f. Dissertação (Mestrado em Engenharia Florestal). Universidade Federal do Paraná.

BOEGER, M. R. T. Morfologia foliar e aspectos nutricionais de espécies arbóreas em três estádios sucessionais, de Floresta Ombrófila Densa das Terras Baixas, Paranaguá, PR. Curitiba, 2000. 150 f. Tese (Doutorado),Curso de Engenharia Florestal da Universidade Federal do Paraná.

DICKOW, K. M. C. Influência estacional e sucessional na lixiviação de nutrientes de serapilheira de Floresta Ombrófila Densa de Terras Baixas. Curitiba, 2000. Monografia apresentada ao Programa especial de Treinamento do Curso de engenharia Florestal da Universidade Federal do Paraná.

IAPAR - INSTITUTO AGRONÔMICO DO PARANÁ. Cartas climáticas básicas do Estado do Paraná. Londrina, 1978, 41p.

MORAES, R. M. Ciclagem de nutrientes minerais em mata atlântica de encosta $e$ mata sobre restinga, na Ilha do Cardoso, Cananéia, SP: produção de serapilheira e transferência de nutrientes. 1993. 151 f. Dissertação (Mestrado em Ecologia Geral) Instituto de Biociências, Universidade de São Paulo.

SPURR, S. H. \& BARNES, V. B. Ecologia Florestal. México: A.G.T. Editora S. A 1982, 690 p.

VITOUSEK, P. M. Litterfall, nutrient cycling and nutrient limitation in tropical forests. 
Ecology. Durhan, 1984. V. 65, n. 1p. 285 298.

WISNIEWSKI, C. Caracterização do ecossistema e estudo das relações solo cobertura vegetal em planície pleistocênica do litoral paranaense. Projeto Integrado - CNPq. Curitiba, 1997. 55p. 